\title{
Relação entre a microfauna de foraminíferos e a granulometria do sedimento do Complexo Recifal de Abrolhos, Bahia, a partir de análises multivariadas
}

\author{
Altair de Jesus Machado ${ }^{1 *}$ \& Helisângela Acris Borges de Araújo ${ }^{2}$
}

\begin{abstract}
Resumo O presente trabalho tem como objetivo identificar, por meio de análises multivariadas, assembleias de foraminíferos que apresentem distribuição influenciada pela granulometria do sedimento. Para tanto, foram analisadas 38 amostras de sedimento superficial de fundo do Complexo Recifal de Abrolhos, na Bahia, com base em critérios sedimentológicos e faunísticos. A análise sedimentológica considerou o percentual das classes texturais cascalho, areia e lama. A análise faunística foi realizada a partir da triagem e da identificação dos 300 primeiros foraminíferos. A partir desses dados, foram realizadas análises multivariadas de classificação e ordenação. A análise de classificação das amostras, com base nos teores de cascalho, areia e lama, revelou a formação de dois grupos (I e II) e quatro subgrupos (IA, IB, IIC e IID), caracterizados pelo predomínio das frações sedimentológicas específicas e confirmados através da análise de ordenação. A análise de classificação das espécies constantes evidenciou a separação de Archaias angulatus dos três agrupamentos microfaunísticos formados (assembleias I, II e III). A correlação entre os agrupamentos sedimentológicos e microfaunísticos permitiu identificar a formação de diferentes assembleias, constituídas a partir de espécies cuja distribuição na área revelou influência direta com o padrão textural do sedimento.
\end{abstract}

Palavras-chave: foraminíferos; granulometria; análises multivariadas.

\begin{abstract}
Relationship between the foraminifera microfauna and the sediment texture from the Abrolhos reef complex based on multivariate analyses. The main goal of this work was to identify, through multivariate analyses, foraminifera assemblages that present distribution influenced by the sediment grain size. Thirty-eight sediment samples from the bottom of the inter-reefal area of Abrolhos were examined for texture and foraminifera composition. The gravel, sand, and mud percentages were calculated. The faunistic analysis was carried out from the identification of the first 300 foraminifera. From these data, multivariate analyses of classification and ordination were performed. The classification analysis of the samples, based on the percentage of gravel, sand, and mud, revealed the presence of two groups (I and II) and four sub-groups (IA, IB, IIC and IID), which are characterized by the dominance of specific grain size fractions, and they were confirmed by the ordination analysis. The classification analysis of the foraminifera species showed the separation of the Archaias angulatus from the three micro-faunistic assemblages (I, II and III). The correlation among the sediment grain size groups and the micro-fauna ones identified the formation of different foraminifera assemblages composed by species whose distribution in the area is directly regulated by the textural character of the sediment.
\end{abstract}

Keywords: foraminifera; sediment grain size; multivariate analyses.

\section{INTRODUÇÃO}

Os foraminíferos são organismos unicelulares inseridos no Reino Protoctista, no Filo Granuloreticulosa e na Classe Foraminiferida (Sen Gupta 1999). Embora ocorram também em ambientes transicionais e dulciaquícolas, a maior diversidade do grupo está associada aos oceanos, no qual ocupam praticamente todos os hábitats. As formas bentônicas são encontradas em todos os ambientes estuarinos, lagunares e marinhos (Debenay et al. 1996), geralmente compondo a epifauna e a infauna do substrato. Os foraminíferos planctônicos, por sua vez, são exclusivamente marinhos, vivem em águas limpas e, em geral, estão próximos à superfície, entre 6 e $30 \mathrm{~m}$ da coluna d'água. A utilização desses organismos em trabalhos que avaliam as condições apresentadas pelos ecossistemas é possível pelo fato de os foraminíferos apresentarem um conjunto de características que os torna importantes em estudos desta natureza. Dentre estes, destacam-se a notável abundância, a facilidade de coleta e a sensibilidade do grupo a alterações nos parâmetros abióticos e bióticos, incluindo luz, temperatura, salinidade, disponibilidade de oxigênio, alcalinidade do meio, profundidade, tipo do substrato e turbidez da água (Murray 1991, Boltovskoy et al. 1991). Alterações em qualquer destes fatores são expressas pelos foraminíferos, principalmente, por meio da variabilidade intraespecífica ou de mudanças na composição das espécies (Debenay 1988, Murray 1991, Bergue \& Coimbra 2008).

O estudo dos foraminíferos em ambientes recifais compreende em trabalhos de importância 
sedimentológica, taxonômica e na distribuição de espécies com base em diferentes parâmetros ambientais. A partir de estudos realizados neste tipo de ecossistema, no Estado da Bahia, tem sido possível estabelecer assembleias (Araújo 2004) e identificar espécies que apresentam distribuição intrinsecamente relacionada com a granulometria do sedimento (Moraes 2001, Silva 2004, Bruno 2008, Moraes 2006, Araújo 2009). Portanto, o presente trabalho teve como objetivo identificar, por meio das análises multivariadas, a relação existente entre a microfauna de foraminíferos e a granulometria do sedimento no complexo recifal de Abrolhos, no extremo Sul do Estado da Bahia.

\section{CARACTERIZAÇÃO DA ÁREA DE ESTUDO}

A área de estudo compreende o trecho da plataforma continental do Estado da Bahia, que se estende desde a cidade de Corumbau até Nova Viçosa, na Bahia, abrangendo estruturas e bancos recifais presentes desde as proximidades da costa até a isóbata de $30 \mathrm{~m}$, entre latitudes de $16^{\circ} 52^{\prime} 58^{\prime \prime} \mathrm{S}$ e $18^{\circ} 06^{\prime} 7,8^{\prime \prime} \mathrm{S}$ e longitudes de $38^{\circ} 16^{\prime} 22,2^{\prime \prime} \mathrm{W}$ e $39^{\circ} 35^{\prime} 26^{\prime \prime}$ W (Fig. 1).

$\mathrm{O}$ clima desta região é tropical úmido tipo $\mathrm{AF}$ de Köppen (SEI 1998, Ocean Wheather 2008), com temperatura média do ar oscilando de 28 a $30^{\circ} \mathrm{C}$, no verão, e 20 a $22{ }^{\circ} \mathrm{C}$, no inverno. A temperatura das águas da superfície do mar varia entre 25 a $27^{\circ} \mathrm{C}$, durante o verão, e 22 a $24{ }^{\circ} \mathrm{C}$, no inverno, apresentando fracos gradientes verticais, enquanto a salinidade varia de 36,5 a 37,0 (Olavo et al. 2007). A média anual de precipitação na região costeira adjacente à área de Abrolhos é de $1.750 \mathrm{~mm}$, sendo março, abril e maio os meses mais chuvosos, concentrando $37 \%$ de toda a precipitação anual, com 612 mm (Leão 1996, Leão et al. 2001).

Formações recifais Em frente à ponta de Corumbau, estão os Recifes de Itacolomís (Fig. 1), que são constituídos por bancos recifais isolados, separados entre si por canais irregulares, com profundidades que variam entre 10 e $20 \mathrm{~m}$ (Fig. 1). Entre a ponta de Corumbau e a cidade do Prado, existem estruturas recifais, estreitas e alongadas, dentre as quais destacam-se os recifes de Tauá, Patachos, Barra do Cahy, Ponta do Mereira, Cumuruxatiba e Calções de Fora, localizados em profundidades de até $15 \mathrm{~m}$ (Fig. 1).

Quase em frente à cidade do Prado, a plataforma alarga-se bruscamente, formando o Banco de Abrolhos, no qual estão os mais conhecidos recifes de corais do Sul da Bahia. Estes recifes espalham-se por uma área de $6.000 \mathrm{~km}^{2}$, formando dois arcos recifais: o arco interno (Fig. 1), paralelo à costa, é constituído por colunas recifais isoladas, em forma de chapeirões, e por plataformas recifais com extensão aproximada de $60 \mathrm{~km}$, estendendo-se desde os recifes em frente à cidade do Prado até aqueles em frente à Nova Viçosa; e o externo (Fig. 1), bordejando a costa Leste das ilhas do Arquipélago de Abrolhos, formado por chapeirões isolados, em águas com profundidades médias de 15 a $20 \mathrm{~m}$ (Leão 1983, Leão et al. 2008).

MATERIAIS E MÉTODOS As amostras utilizadas foram coletadas durante uma expedição organizada pelo Instituto de Conservação Internacional do Brasil, como parte do programa de avaliação do Banco de Abrolhos e foram fornecidas para o estudo de foraminíferos pela professora Zelinda Leão, da Universidade Federal da Bahia (UFBA).

Durante novembro e dezembro de 2000, foram coletadas 68 amostras de sedimento superficial de fundo, abrangendo recifes que se estendem desde a linha de costa, entre as cidades de Corumbau e Nova Viçosa, até a isóbata de $30 \mathrm{~m}$. Do total de 68 amostras, apenas 38 foram selecionadas para a realização deste trabalho, abrangendo-se as formações recifais da área.

Em cada estação, coletou-se, na base das estruturas recifais (em profundidades de 1,8 a $29 \mathrm{~m}$ ), uma média de $400 \mathrm{~g}$ de sedimento, destinado à análise granulométrica, análise do teor de carbonato, determinação dos componentes biogênicos e triagem dos foraminíferos. Porém, neste trabalho, somente serão apresentados e discutidos os dados granulométricos e faunísticos.

No Laboratório de Estudos Costeiros do Instituto de Geociências da UFBA, realizou-se a lavagem das amostras em peneira, com espaçamento de $0,062 \mathrm{~mm}$, para a eliminação dos sais. Em seguida, separou-se $2 / 3$ do peso total de cada amostra para a análise dos parâmetros sedimentológicos, que foi realizada por meio do estudo de caracterização do sedimento de fundo na área de Abrolhos, por Leão et al. (2001), permitindo-se classificar os sedimentos nos intervalos de classe cascalho $(>2 \mathrm{~mm})$, areia (entre 2 e $0,062 \mathrm{~mm}$ ) e lama $(<0,062 \mathrm{~mm})$, como pode ser visto na Tab. 1, além de avaliar o teor de grãos carbonáticos e siliciclásticos em cada amostra (Tab. 2). A porção restante (1/3) de cada amostra foi reservada à análise da microfauna de foraminíferos, 


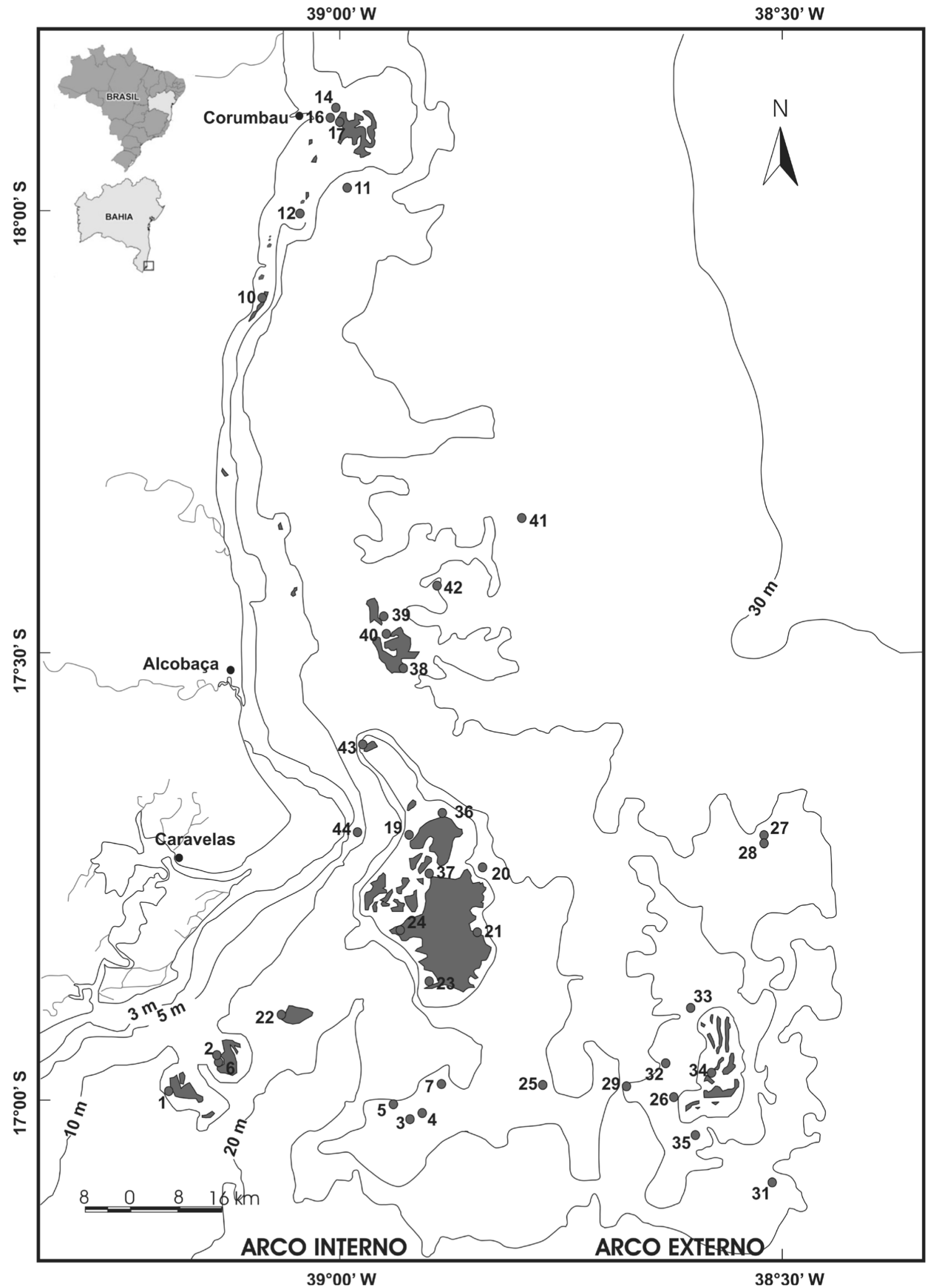

Figura 1 - Mapa de localização das estações de coleta nas áreas inter-recifais estudadas (modificado de Leão et al. 2001).

sendo distribuídas em bandejas, colocados para secar ao ar livre e acondicionados em sacos plásticos previamente etiquetados.

Para a determinação da diversidade faunística de foraminíferos, nas estações amostradas, realizou-se, sob microscópio estereoscópio, o isolamento e o armazenamento em lâminas de Franke, dos 300 primeiros foraminíferos (bentônicos e planctônicos) encontrados em cada amostra, sem que tenha sido realizado peneiramento. Quando necessário, devido à grande quantidade de material, as amostras foram quarteadas. 
Tabela 1 - Percentuais das frações granulométricas cascalho (>2 mm), areia (entre $2 \mathrm{~mm}$ e 0,062 $\mathrm{mm}$ ) e lama $(<0,062 \mathrm{~mm})$ nas amostras

\begin{tabular}{|c|c|c|c|}
\hline Amostras & Cascalho & Areia & Lama \\
\hline 1 & 12,73 & 76,36 & 10,91 \\
\hline 2 & 0,05 & 82,44 & 17,51 \\
\hline 3 & 4,79 & 48,22 & 46,99 \\
\hline 4 & 1,12 & 21,10 & 77,78 \\
\hline 5 & 0,74 & 16,05 & 83,21 \\
\hline 6 & 0,19 & 47,38 & 52,43 \\
\hline 7 & 5,88 & 31,88 & 62,24 \\
\hline 10 & 15,11 & 61,36 & 23,53 \\
\hline 11 & 13,28 & 78,25 & 8,47 \\
\hline 12 & 0,06 & 75,88 & 24,06 \\
\hline 14 & 7,00 & 48,95 & 44,05 \\
\hline 16 & 27,34 & 45,60 & 27,06 \\
\hline 17 & 0,18 & 4,62 & 95,20 \\
\hline 19 & 27,88 & 71,77 & 0,35 \\
\hline 20 & 2,15 & 56,05 & 41,80 \\
\hline 21 & 7,05 & 58,12 & 34,83 \\
\hline 22 & 3,59 & 95,82 & 0,59 \\
\hline 23 & 0,11 & 21,86 & 78,03 \\
\hline 24 & 6,89 & 90,44 & 2,67 \\
\hline 25 & 36,49 & 60,92 & 2,59 \\
\hline 26 & 47,78 & 49,28 & 2,94 \\
\hline 27 & 66,69 & 31,63 & 1,68 \\
\hline 28 & 17,62 & 72,96 & 9,42 \\
\hline 29 & 49,31 & 50,29 & 0,40 \\
\hline 31 & 13,88 & 80,26 & 5,86 \\
\hline 32 & 3,89 & 95,76 & 0,35 \\
\hline 33 & 1,06 & 71,38 & 27,56 \\
\hline 34 & 9,63 & 14,89 & 75,48 \\
\hline 35 & 0,22 & 26,41 & 73,37 \\
\hline 36 & 5,98 & 35,46 & 58,56 \\
\hline 37 & 8,42 & 69,41 & 22,17 \\
\hline 38 & 1,25 & 11,42 & 87,33 \\
\hline 39 & 1,92 & 45,89 & 52,19 \\
\hline 40 & 0,04 & 8,96 & 91,00 \\
\hline 41 & 38,69 & 40,62 & 20,69 \\
\hline 42 & 5,72 & 74,06 & 20,22 \\
\hline 43 & 2,30 & 41,41 & 56,29 \\
\hline 44 & 0,00 & 36,60 & 63,40 \\
\hline
\end{tabular}

Os foraminíferos retirados de cada amostra foram identificados sob a lupa binocular, com base principalmente nos trabalhos de Andrade (1997), Barker (1960), Bock (1971), Boltovskoy et al. (1980), Closs \& Barberena (1960), Leipnitz (1991), Leipnitz et al. (1992), Levy et al. (1995), Machado (1981), Rossi (1999) e Tinoco (1955, 1958).

A partir dos dados obtidos com a análise da fauna de foraminíferos (número de indivíduos por espécie) e seguindo a metodologia proposta por Dajoz (1983), Tinoco (1989), Clarke \& Warwick (1994) e Valentin (2000), foram calculados os índices de
Tabela 2 - Percentuais de grãos carbonáticos e siliciclásticos nas amostras

\begin{tabular}{|c|c|c|}
\hline Amostras & Carbonáticos & Siliciclásticos \\
\hline 1 & 33,49 & 66,51 \\
\hline 2 & 92,80 & 7,20 \\
\hline 3 & 93,78 & 6,22 \\
\hline 4 & 98,10 & 1,90 \\
\hline 5 & 93,14 & 6,86 \\
\hline 6 & 57,01 & 42,99 \\
\hline 7 & 90,54 & 9,46 \\
\hline 10 & 73,17 & 26,83 \\
\hline 11 & 98,77 & 1,23 \\
\hline 12 & 89,64 & 10,36 \\
\hline 14 & 100,00 & 0,00 \\
\hline 16 & 80,19 & 19,81 \\
\hline 17 & 100,00 & 0,00 \\
\hline 19 & 97,56 & 2,44 \\
\hline 20 & 74,90 & 25,10 \\
\hline 21 & 91,91 & 8,09 \\
\hline 22 & 90,99 & 9,01 \\
\hline 23 & 93,89 & 6,11 \\
\hline 24 & 98,18 & 1,82 \\
\hline 25 & 98,67 & 1,33 \\
\hline 26 & 96,08 & 3,92 \\
\hline 27 & 97,17 & 2,83 \\
\hline 28 & 94,79 & 5,21 \\
\hline 29 & 95,75 & 4,25 \\
\hline 31 & 100,00 & 0,00 \\
\hline 32 & 47,64 & 52,36 \\
\hline 33 & 97,73 & 2,27 \\
\hline 34 & 100,00 & 0,00 \\
\hline 35 & 99,04 & 0,96 \\
\hline 36 & 88,19 & 11,81 \\
\hline 37 & 99,12 & 0,88 \\
\hline 38 & 99,06 & 0,94 \\
\hline 39 & 98,36 & 1,64 \\
\hline 40 & 94,51 & 5,49 \\
\hline 41 & 97,61 & 2,39 \\
\hline 42 & 76,89 & 23,11 \\
\hline 43 & 85,27 & 14,73 \\
\hline 44 & 11,89 & 88,11 \\
\hline
\end{tabular}

abundância relativa (AR) e frequência de ocorrência, além de terem sido realizadas análises multivariadas.

A AR das espécies foi obtida pela fórmula $A R$ $=\mathrm{n} \times 100 / \mathrm{T}$, em que " $\mathrm{n}$ " representa o número de indivíduos de uma determinada espécie e "T" significa o número total de indivíduos da amostra (Tinoco 1989). A frequência de ocorrência, calculada pela fórmula $\mathrm{FO}=\mathrm{p} \times 100 / \mathrm{P}$, representa a relação entre o número de amostras no qual a espécie ocorreu (p) e o número total de amostras analisadas (P). Considerando os valores de frequência de ocorrência, as espécies foram classificadas, segundo Dajoz (1983), em constantes (presentes em mais de 50\% das amostras), acessórias 
(de 25 a $50 \%$ das amostras) ou acidentais (menos de $25 \%$ das amostras).

Os dados quantitativos (abundância absoluta das espécies e variáveis sedimentológicas) foram submetidos às análises multivariadas de classificação ou agrupamento (Análises de Cluster), possibilitando reconhecer o grau de similaridade entre as variáveis analisadas. Desta forma, utilizando-se o programa MVSP (versão 3.11), esta análise foi efetuada sob dois diferentes modos: Q (agrupamento entre amostras, segundo as espécies nelas contidas e segundo os teores sedimentológicos presentes em cada amostra) e R (agrupamento entre espécies, considerando as amostras que as encerram). Para quantificar a similaridade entre os objetos, utilizou-se o coeficiente de similaridade de Bray-Curtis.

Visando confirmar o agrupamento feito entre as amostras, realizou-se a análise de ordenação (multidimensional scaling - MDS), frequentemente aplicada em dados ecológicos, como cita Clarke \& Warwick (1994). Para realizar esta análise, foram utilizados os programas Statistics Basic (Edição 1999) e MVSP (versão 3.11), por meio da aplicação do coeficiente de similaridade de Bray-Curtis.

RESULTADOSDentre as amostras, registrou-se o domínio das frações areia e lama. A fração areia está representada em todas as estações, predominando com percentuais acima de $50 \%$ em quase metade delas. A fração lama também está presente em todas as estações, destacando-se em 14 amostras com representatividade superior a 50\%. A fração cascalho é menos representativa, estando presente com percentuais acima de $50 \%$ apenas na amostra 27 e acima de $30 \%$ em outras quatro amostras (Tab. 1 e Fig. 2).

A análise da composição do sedimento revelou que os siliciclásticos estão presentes em quase todas as amostras, estando ausentes apenas nas estações 14, 17, 31 e 34 . A ocorrência de sedimento siliciclástico com teor superior a $10 \%$ foi verificada em 11 amos$\operatorname{tras}(1,6,10,12,16,20,32,36,42,43$ e 44), as quais, com exceção de amostra 32, situam-se ao redor dos recifes costeiros (arco interno), como observado na Fig. 1. As demais amostras, cujo teor de sedimento siliciclástico está entre 0 e $10 \%$, encontram-se dispersas pela área de estudo. Os sedimentos carbonáticos são os componentes dominantes da fração fina do sedimento. Com exceção das amostras 1, 6, 10, 20, 32,42 e 44 , este sedimento constitui mais de $80 \%$ da composição das amostras analisadas, estando distribuídos ao longo de toda a área.
As microfaunas bentônica e planctônica de foraminíferos são compostas por 150 espécies, compreendendo 40 gêneros (38 bentônicos e 2 planctônicos), 18 superfamílias e 5 subordens, segundo a classificação sistemática proposta por Loeblich \& Tappan (1988). Dentre as ordens encontradas, a ordem Miliolina apresenta maior número de espécies (99), seguida pelas ordens Rotaliina (39), Textulariina (9), Globigerinina (2) e Lagenina (1). A ordem Miliolina, além de apresentar maior número de representantes, é a mais representativa em número de indivíduos (8.108 espécimes), seguida pelas ordens Rotaliina (3024), Textulariina (264), Globigeriina (2) e Lagenina (2).

A análise dos dados obtidos a partir do cálculo da frequência de ocorrência das 150 espécies identificadas evidencia a existência de 32 espécies consideradas constantes (21,33\%), 27 acessórias (18,00\%) e 91 acidentais (60,67\%), como obervado na Tab. 3 .

Entre as espécies consideradas constantes, não foram observados indivíduos com frequência de ocorrência em $100 \%$ das amostras. A espécie Quinqueloculina lamarckiana apresenta a maior frequência de ocorrência da área (97,37\%), estando presente em 37 amostras, das 38 analisadas. A espécie constante que apresenta a menor frequência de ocorrência é Spiroloculina caduca (52,63\%), presente em 20 amostras.

As espécies Bigenerina sp2, Cibicides sp1, Dentostomina enoplastona, Gypsina vesicularis e Peneroplis pertusus var. discoideus são, entre as consideradas acessórias, as que apresentam menor frequência de ocorrência (26,32\%), estando presentes em dez amostras. As espécies acessórias com maior frequência são Triloculina sommerii (47,37\%) e Triloculina tricarinata (47,37\%), presentes em 18 amostras.

Entre as espécies consideradas acidentais, Cibicides refulgens, Clavulina tricarinata, Miliolinella labiosa, Quinqueloculina cuveriana, Quinqueloculina laevigata e Quinqueloculina sp4 são as de maior frequência $(23,68 \%)$, sendo encontradas em nove amostras. Por outro lado, $34,06 \%$ das espécies acidentais, Articulina atlantica, Bigenerina sp 1, Bolivina tortuosa, Bolivina sp, Clavulina angularis, Discorbis bertheloti, Discorbis bertheloti var. floridensis, Discorbis candeiana, Elphidium galvestonense, Globigerinoides ruber, Hanzawaia bertheloti, Massilina asperula, Oolina sp., Orbulinauniversa,Planorbulinaacervalis, Pyrgorigens, Quinqueloculina bosciana, Quinqueloculina bradyana, Quinqueloculina collumnosa, Quinqueloculina lata, Quinqueloculina pseudoreticulata, Quinqueloculina 
Tabela 3 - Número de amostras em que cada espécie ocorreu; frequência de ocorrência (FO); abundância absoluta (AA) e abundância relativa (AR)

\begin{tabular}{|c|c|c|c|c|}
\hline Espécies & $\begin{array}{l}\text { Número de } \\
\text { amostras }\end{array}$ & FO & AA & $\operatorname{AR}(\%)$ \\
\hline Ammonia beccarii & 23 & 0,00 & 475 & 4,17 \\
\hline Amphistegina gibbosa & 23 & 60,53 & 136 & 1,19 \\
\hline Amphistegina lessonii & 31 & 81,58 & 414 & 3,63 \\
\hline Amphistegina radiata & 8 & 21,05 & 24 & 0,21 \\
\hline Archaias angulatus & 27 & 71,05 & 1565 & 13,73 \\
\hline Articulina antillarum & 8 & 21,05 & 25 & 0,22 \\
\hline Articulina atlantica & 1 & 2,63 & 1 & 0,01 \\
\hline Articulina mucronata & 4 & 10,53 & 6 & 0,05 \\
\hline Articulina multilocularis & 6 & 15,79 & 20 & 0,18 \\
\hline Articulina pacifica & 4 & 10,53 & 8 & 0,07 \\
\hline Bigenerina nodosaria & 5 & 13,16 & 29 & 0,25 \\
\hline Bigenerina rephandus & 1 & 2,63 & 6 & 0,05 \\
\hline Bigenerina $s p$ & 10 & 26,32 & 69 & 0,61 \\
\hline Bolivina pulchella & 4 & 10,53 & 7 & 0,06 \\
\hline Bolivina tortuosa & 1 & 2,63 & 1 & 0,01 \\
\hline Bolivina sp & 1 & 2,63 & 1 & 0,01 \\
\hline Borelis pulcha & 14 & 36,84 & 140 & 1,23 \\
\hline Cibicides akmerianus & 2 & 5,26 & 11 & 0,10 \\
\hline Cibicides pseudogenerianus & 4 & 10,53 & 7 & 0,06 \\
\hline Cibicides refulgens & 9 & 23,68 & 28 & 0,25 \\
\hline Cibicides repandus & 10 & 26,32 & 39 & 0,34 \\
\hline Cibicides $s p$ & 2 & 5,26 & 2 & 0,02 \\
\hline Clavulina angularis & 1 & 2,63 & 1 & 0,01 \\
\hline Clavulina tricarinata & 9 & 23,68 & 28 & 0,25 \\
\hline Cornuspira planorbis & 8 & 21,05 & 27 & 0,24 \\
\hline Dentostomina antillarum & 6 & 15,79 & 9 & 0,08 \\
\hline Dentostomina enoplastoma & 10 & 26,32 & 29 & 0,25 \\
\hline Discorbis bertheloti & 1 & 2,63 & 2 & 0,02 \\
\hline Discorbis bertheloti var, floridensis & 1 & 2,63 & 1 & 0,01 \\
\hline Discorbis candeiana & 1 & 2,63 & 12 & 0,11 \\
\hline Discorbis floridana & 24 & 63,16 & 69 & 0,61 \\
\hline Discorbis mira & 26 & 68,42 & 100 & 0,88 \\
\hline Discorbis williamsoni & 2 & 5,26 & 9 & 0,08 \\
\hline Elphidium discoidale & 28 & 73,68 & 287 & 2,52 \\
\hline Elphidium galvestonense & 1 & 2,63 & 7 & 0,06 \\
\hline Elphidium morenoi & 8 & 21,05 & 45 & 0,39 \\
\hline Elphidium poeyanum & 29 & 76,32 & 560 & 4,91 \\
\hline Elphidium sagrum & 23 & 60,53 & 75 & 0,66 \\
\hline Eponides antillarum & 8 & 21,05 & 25 & 0,22 \\
\hline Eponides repandus & 23 & 60,53 & 172 & 1,51 \\
\hline Globigerinoides ruber & 1 & 2,63 & 1 & 0,01 \\
\hline Gypsina vesicularis & 10 & 26,32 & 21 & 0,18 \\
\hline Hanzawaia bertheloti & 1 & 2,63 & 1 & 0,01 \\
\hline Hauerina involuta & 2 & 5,26 & 2 & 0,02 \\
\hline Heterostegina depressa & 21 & 55,26 & 81 & 0,71 \\
\hline Heterostegina suborbicularis & 3 & 7,89 & 4 & 0,04 \\
\hline Homotrema rubrum & 14 & 36,84 & 111 & 0,97 \\
\hline Massilina asperula & 1 & 2,63 & 1 & 0,01 \\
\hline Massilina pernambucensis & 2 & 5,26 & 2 & 0,02 \\
\hline Miliolinella labiosa & 9 & 23,68 & 11 & 0,10 \\
\hline Miliolinella suborbicularis & 11 & 28,95 & 31 & 0,27 \\
\hline Miliolinella subrotunda & 17 & 44,74 & 55 & 0,48 \\
\hline Nonion grateloupi & 15 & 39,47 & 80 & 0,70 \\
\hline Nonion $s p$ & 2 & 5,26 & 3 & 0,03 \\
\hline
\end{tabular}


Tabela-Continuação

\begin{tabular}{|c|c|c|c|c|}
\hline Nonionella atlantica & 6 & 15,79 & 7 & 0,06 \\
\hline Oolina $s p$ & 1 & 2,63 & 2 & 0,02 \\
\hline Orbulina universa & 1 & 2,63 & 1 & 0,01 \\
\hline Peneroplis bradyi & 19 & 50,00 & 93 & 0,82 \\
\hline Peneroplis carinatus & 25 & 65,79 & 320 & 2,81 \\
\hline Peneroplis pertusus & 14 & 36,84 & 51 & 0,45 \\
\hline Peneroplis pertusus var. discoideus & 10 & 26,32 & 23 & 0,20 \\
\hline Peneroplis proteus & 21 & 55,26 & 105 & 0,92 \\
\hline Planorbulina acervalis & 1 & 2,63 & 1 & 0,01 \\
\hline Poroeponides lateralis & 15 & 39,47 & 70 & 0,61 \\
\hline Pyrgo bulloides & 31 & 81,58 & 270 & 2,37 \\
\hline Pyrgo comata & 7 & 18,42 & 31 & 0,27 \\
\hline Pyrgo denticulata & 13 & 34,21 & 43 & 0,38 \\
\hline Pyrgo elongata & 31 & 81,58 & 192 & 1,68 \\
\hline Pyrgo patagonica & 16 & 42,11 & 45 & 0,39 \\
\hline Pyrgo rigens & 1 & 2,63 & 2 & 0,02 \\
\hline Pyrgo subsphaerica & 34 & 89,47 & 375 & 3,29 \\
\hline Pyrgo tainanensis & 7 & 18,42 & 24 & 0,21 \\
\hline Quinqueloculina agglutinans & 5 & 13,16 & 7 & 0,06 \\
\hline Quinqueloculina angulata & 27 & 71,05 & 281 & 2,46 \\
\hline Quinqueloculina bicarinata & 16 & 42,11 & 64 & 0,56 \\
\hline Quinqueloculina bicornis & 28 & 73,68 & 93 & 0,82 \\
\hline Quinqueloculina bicostata & 23 & 60,53 & 167 & 1,46 \\
\hline Quinqueloculina bosciana & 1 & 2,63 & 12 & 0,11 \\
\hline Quinqueloculina bradyana & 1 & 2,63 & 1 & 0,01 \\
\hline Quinqueloculina candeiana & 29 & 76,32 & 253 & 2,22 \\
\hline Quinqueloculina carinata & 2 & 5,26 & 6 & 0,05 \\
\hline Quinqueloculina collumnosa & 1 & 2,63 & 1 & 0,01 \\
\hline Quinqueloculina costata & 3 & 7,89 & 4 & 0,04 \\
\hline Quinqueloculina kerimbatica & 8 & 21,05 & 23 & 0,20 \\
\hline Quinqueloculina cuveriana & 9 & 23,68 & 19 & 0,17 \\
\hline Quinqueloculina disparilis curta & 35 & 92,11 & 606 & 5,32 \\
\hline Quinqueloculina elongata & 9 & 23,68 & 20 & 0,18 \\
\hline Quinqueloculina funafutiensis & 3 & 7,89 & 4 & 0,04 \\
\hline Quinqueloculina granulocostata & 8 & 21,05 & 9 & 0,08 \\
\hline Quinqueloculina horrida & 3 & 7,89 & 4 & 0,04 \\
\hline Quinqueloculina laevigata & 9 & 23,68 & 28 & 0,25 \\
\hline Quinqueloculina lamarckiana & 37 & 97,37 & 914 & 8,02 \\
\hline Quinqueloculina lata & 1 & 2,63 & 1 & 0,01 \\
\hline Quinqueloculina magoi & 5 & 13,16 & 22 & 0,19 \\
\hline Quinqueloculina parkeri & 24 & 63,16 & 188 & 1,65 \\
\hline Quinqueloculina philipensis & 11 & 28,95 & 13 & 0,11 \\
\hline Quinqueloculina poeyana & 17 & 44,74 & 85 & 0,75 \\
\hline Quinqueloculina polygona & 36 & 94,74 & 373 & 3,27 \\
\hline Quinqueloculina pseudoreticulata & 1 & 2,63 & 2 & 0,02 \\
\hline Quinqueloculina punctulata & 1 & 2,63 & 1 & 0,01 \\
\hline Quinqueloculina reticulata & 5 & 13,16 & 13 & 0,11 \\
\hline Quinqueloculina seminulum & 5 & 13,16 & 5 & 0,04 \\
\hline Quinqueloculina sulcata & 16 & 42,11 & 62 & 0,54 \\
\hline Quinqueloculina tenuis & 1 & 2,63 & 2 & 0,02 \\
\hline Quinqueloculina tricarinata & 1 & 2,63 & 1 & 0,01 \\
\hline Quinqueloculina venusta & 1 & 2,63 & 1 & 0,01 \\
\hline Quinqueloculina sp 1 & 5 & 13,16 & 13 & 0,11 \\
\hline Quinqueloculina sp 2 & 13 & 34,21 & 65 & 0,57 \\
\hline Quinqueloculina sp 3 & 5 & 13,16 & 9 & 0,08 \\
\hline Schlumbergerina alveoliniformis & 7 & 18,42 & 21 & 0,18 \\
\hline
\end{tabular}


Tabela-Continuação

\begin{tabular}{|c|c|c|c|c|}
\hline Sigmoilopsis sp & 1 & 2,63 & 5 & 0,04 \\
\hline Siphogenerina rephandus & 2 & 5,26 & 29 & 0,25 \\
\hline Siphogenerina $s p$ & 1 & 2,63 & 8 & 0,07 \\
\hline Siphonina echinata & 2 & 5,26 & 6 & 0,05 \\
\hline Siphonina pulchra & 13 & 34,21 & 33 & 0,29 \\
\hline Sorites marginalis & 28 & 73,68 & 321 & 2,82 \\
\hline Spiroloculina antilarum & 24 & 63,16 & 83 & 0,73 \\
\hline Spiroloculina caduca & 20 & 52,63 & 84 & 0,74 \\
\hline Spiroloculina communis & 5 & 13,16 & 9 & 0,08 \\
\hline Spiroloculina stebani & 22 & 57,89 & 53 & 0,46 \\
\hline Spiroloculina profunda & 4 & 10,53 & 4 & 0,04 \\
\hline Spiroloculina $s p$ & 1 & 2,63 & 1 & 0,01 \\
\hline Textularia agglutinans & 22 & 57,89 & 70 & 0,61 \\
\hline Textularia candeiana & 11 & 28,95 & 32 & 0,28 \\
\hline Textularia gramen & 6 & 15,79 & 9 & 0,08 \\
\hline Textularia kerimbaensis & 6 & 15,79 & 11 & 0,10 \\
\hline Triloculina affinis & 4 & 10,53 & 6 & 0,05 \\
\hline Triloculina baldai & 12 & 31,58 & 36 & 0,32 \\
\hline Triloculina bertheliniana & 1 & 2,63 & 1 & 0,01 \\
\hline Triloculina bicarinata & 24 & 63,16 & 66 & 0,58 \\
\hline Triloculina candeiana & 5 & 13,16 & 14 & 0,12 \\
\hline Triloculina caudata & 3 & 7,89 & 7 & 0,06 \\
\hline Triloculina consobrina & 15 & 39,47 & 83 & 0,73 \\
\hline Triloculina elongata & 4 & 10,53 & 4 & 0,04 \\
\hline Triloculina gracilis & 5 & 13,16 & 10 & 0,09 \\
\hline Triloculina laevigata & 4 & 10,53 & 6 & 0,05 \\
\hline Triloculina linneiana & 4 & 10,53 & 7 & 0,06 \\
\hline Triloculina lutea & 2 & 5,26 & 2 & 0,02 \\
\hline Triloculina oblonga & 12 & 31,58 & 55 & 0,48 \\
\hline Triloculina planciana & 3 & 7,89 & 3 & 0,03 \\
\hline Triloculina quadrata & 5 & 13,16 & 9 & 0,08 \\
\hline Triloculina quadrilateralis & 8 & 21,05 & 28 & 0,25 \\
\hline Triloculina reticulata & 6 & 15,79 & 23 & 0,20 \\
\hline Triloculina rotunda & 3 & 7,89 & 3 & 0,03 \\
\hline Triloculina rupertiana & 11 & 28,95 & 19 & 0,17 \\
\hline Triloculina sommerii & 18 & 47,37 & 62 & 0,54 \\
\hline Triloculina subrotunda & 1 & 2,63 & 1 & 0,01 \\
\hline Triloculina tricarinata & 18 & 47,37 & 38 & 0,33 \\
\hline Triloculina trigonula & 26 & 68,42 & 184 & 1,61 \\
\hline Wiesnerella auriculata & 1 & 2,63 & 1 & 0,01 \\
\hline Espécies não identificáveis & 22 & 57,89 & 50 & 0,44 \\
\hline Total & & & 11.400 & 100,00 \\
\hline
\end{tabular}

punctulata, Quinqueloculina tenuis, Quinqueloculina tricarinata, Quinqueloculina venusta, Sigmoilopsis sp, Siphogenerina sp, Spiroloculina sp, Triloculina bertheliniana, Triloculina subrotunda e Wiesnerella auriculata aparecem com frequência de ocorrência de 2,63\%, tendo sido encontradas em apenas uma amostra.

\section{Análises de classificação numérica e ordenação}

Associações sedimentológicas Com base nos teores sedimentológicos de cascalho, areia e lama, construiu-se um dendograma das estações (Fig. 3), que evidencia a associação das amostras por meio do cálculo do coeficiente de similaridade de Bray-Curtis. Neste dendograma, é possível identificar a formação de dois grandes grupos (Grupos I e II) à distância de 0,38. Os Grupos I e II dividem-se em dois subgrupos, estando o Grupo I subdividido nos subgrupos A e B, e o II subdividido nos subgrupos C e D (Fig. 3).

O Grupo I é formado pelas amostras constituídas por sedimento com teor de lama superior a $30 \%$ (Tab. 1). No subgrupo A estão as amostras com 


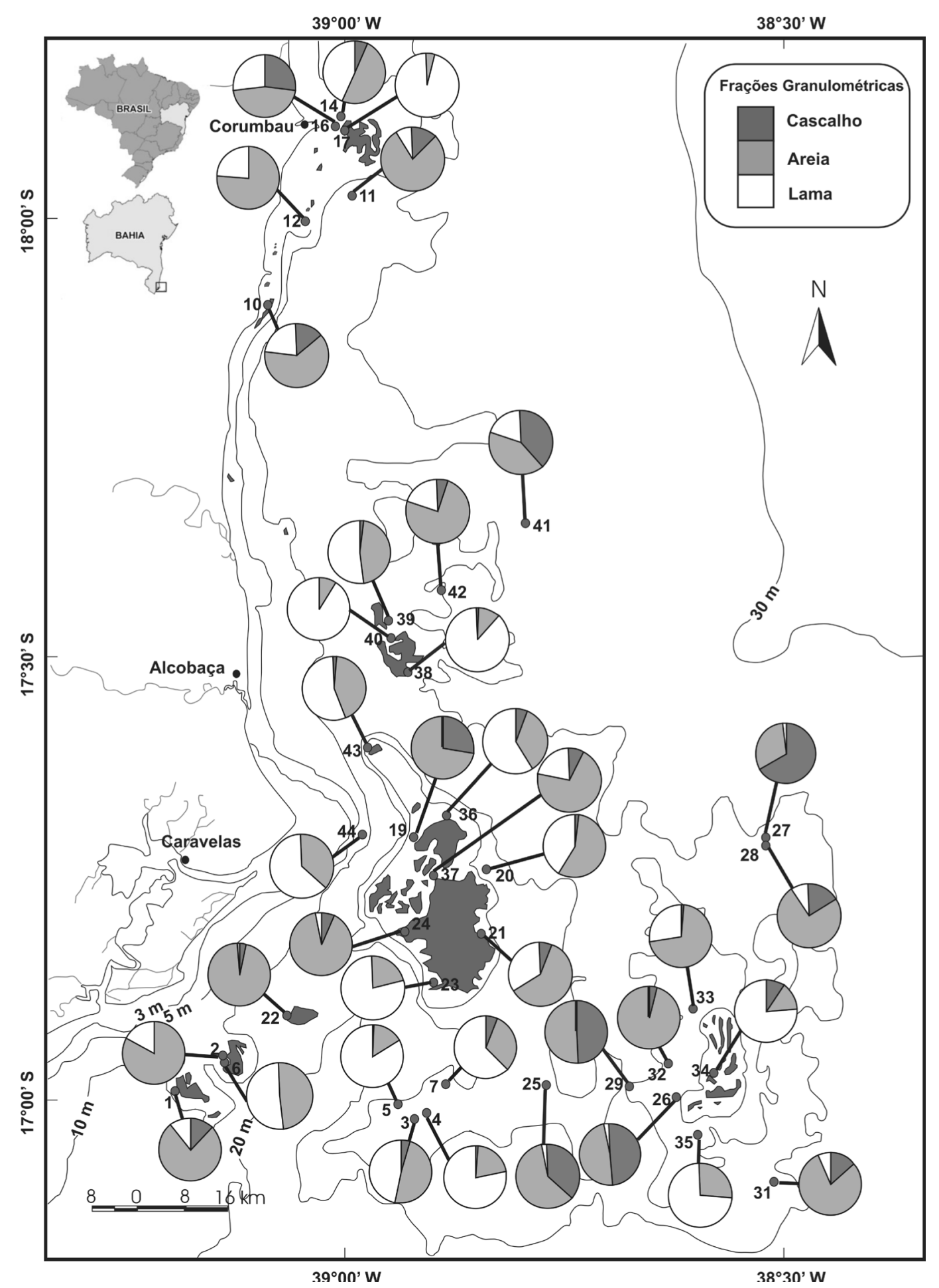

Figura 2 - Distribuição das frações granulométricas cascalho, areia e lama (modificado de Leão et al. 2001).

percentuais de lama superiores a $70 \%$ (Tab. 1). O subgrupo B compreende as amostras que apresentam percentuais de lama entre 30 e $70 \%$ (Tab. 1 e Fig. 3).

O Grupo II é representado pelas amostras que apresentam teores de lama inferiores a 30\% (Tab. 1). Inicialmente, tem-se o subgrupo $C$, formado pelas amostras que possuem percentuais de sedimento tamanho cascalho superiores a $20 \%$ e areia inferiores a $60 \%$ (Tab. 1). O subgrupo $\mathrm{D}$ corresponde à associação formada por amostras com percentuais de sedimento tamanho cascalho inferiores a $20 \%$ e areia superiores a $60 \%$ (Tab. 1 e Fig. 3).

A interpretação efetuada a partir dos dados obtidos com a análise de classificação pôde ser confirmada pela análise de ordenação entre as amostras. A Fig. 4 evidencia o produto desta análise, que demonstrou um valor de estresse $(0,02)$, o que valida seu resultado. Na análise de ordenação também é possível observar que o agrupamento entre as amostras, com base nos valores texturais do sedimento, 
é feito em quatro subgrupos distintos (a, a', b, b'), formados com base nos mesmos critérios utilizados na análise de classificação.

Na Fig. 4 pode-se observar que o subgrupo A é representado pelas mesmas amostras que formam o subgrupo $\mathrm{C}$ da análise de classificação, bem como os subgrupos a', b e b' correspondem aos subgrupos D, $\mathrm{B}$ e A, respectivamente.

Associações faunisticas $\mathrm{O}$ dendograma, representado na Fig. 5, apresenta o resultado do agrupamento das espécies consideradas constantes (frequência de ocorrência superior a 50\%) na área estudada.

Pode ser observada, inicialmente, a nítida separação da espécie Archaias angulatus do agrupamento efetuado. Em seguida, considerando como nível de corte o índice de similaridade de BrayCurtis de 0,7, foi possível evidenciar a formação de três agrupamentos microfaunísticos. A assembleia I é formada por 12 espécies, sendo elas: Quinqueloculina bicostata, Textularia agglutinans,
Triloculina bicarinata, Quinqueloculina bicornis, Peneroplis proteus, Peneroplis bradyi, Elphidium sagrum, Discorbis mira, Spiroloculina caduca, Spiroloculina antilarum, Spiroloculina estebani e Discorbis floridana (Fig. 5). Esta assembleia reúne as espécies que não apresentaram preferência pela característica textural do sedimento. Em geral, estas espécies apresentam-se abundantes em amostras dos Grupos I e II da Fig. 3.

A assembleia II compreende sete espécies: Eponides sp., Quinqueloculina parkeri, Quinqueloculina candeiana, Peneroplis carinatus, Amphistegina lessonii, Heterostegina depressa e Amphistegina gibbosa (Fig. 5). Essa assembleia é formada por espécies que, na grande maioria, apresentam-se mais abundantes nas amostras correspondentes ao Grupo II da Fig. 3, as quais são constituídas por teores de lama inferiores a $30 \%$.

A assembleia III, por sua vez, é composta por 12 espécies: Sorites marginalis, Pyrgo subsphaerica, Pyrgo bulloides, Quinqueloculina lamarckiana,

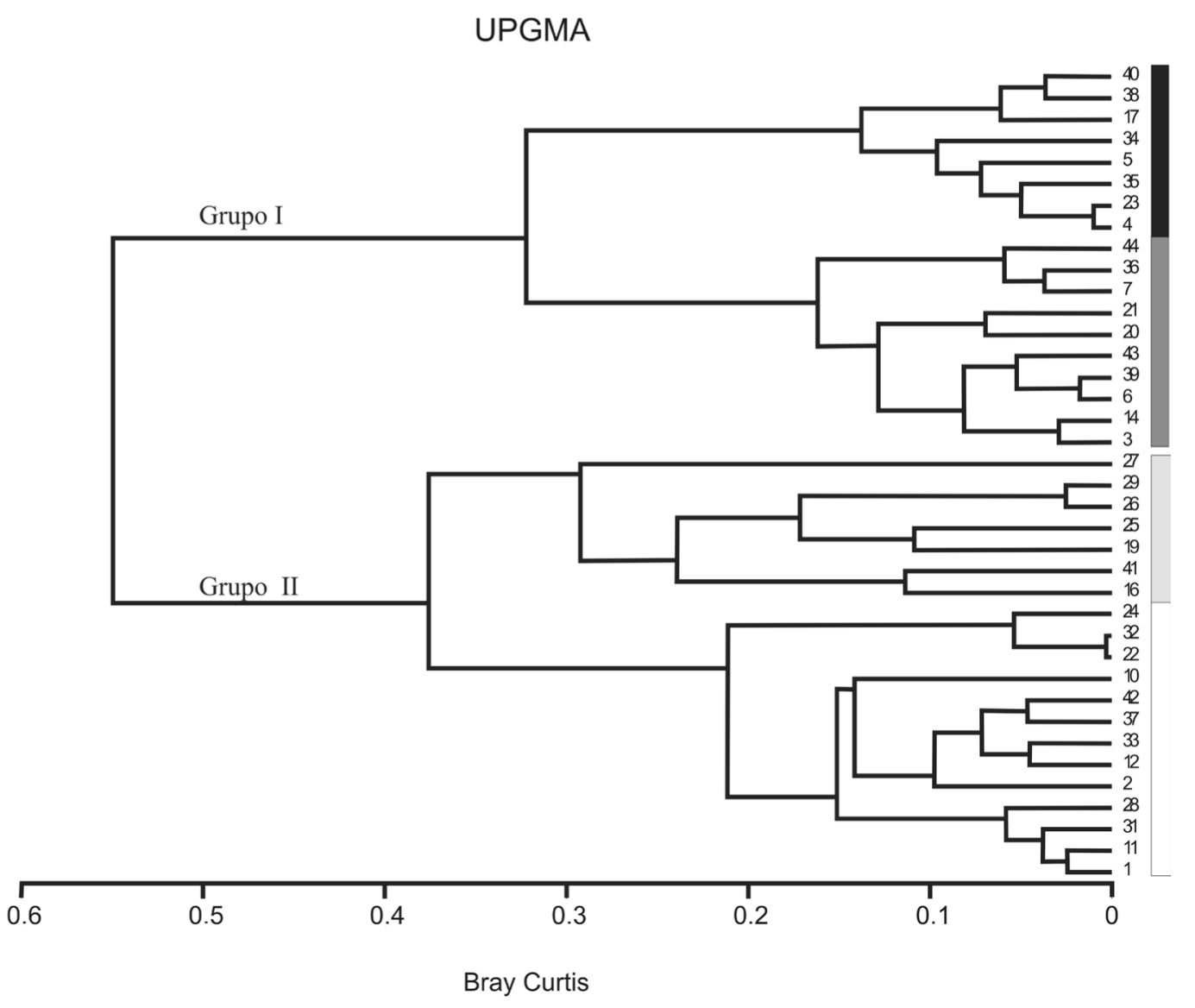

Subgrupo A

Subgrupo B

Subgrupo C

Subgrupo D

Figura 3 - Dendograma representativo do agrupamento das estações a partir dos dados sedimentológicos (percentuais de cascalho, areia e lama). Grupo I =lama $>30 \%$ (Subgrupo A =lama $>70 \%$; Subgrupo B =lama 30 a 70\%); Grupo II = lama $<30 \%$ (Subgrupo $C=$ cascalho $>20 \%$ e areia $<60 \%$; Subgrupo D $=$ cascalho $<20 \%$ e areia $>60 \%$ ). 


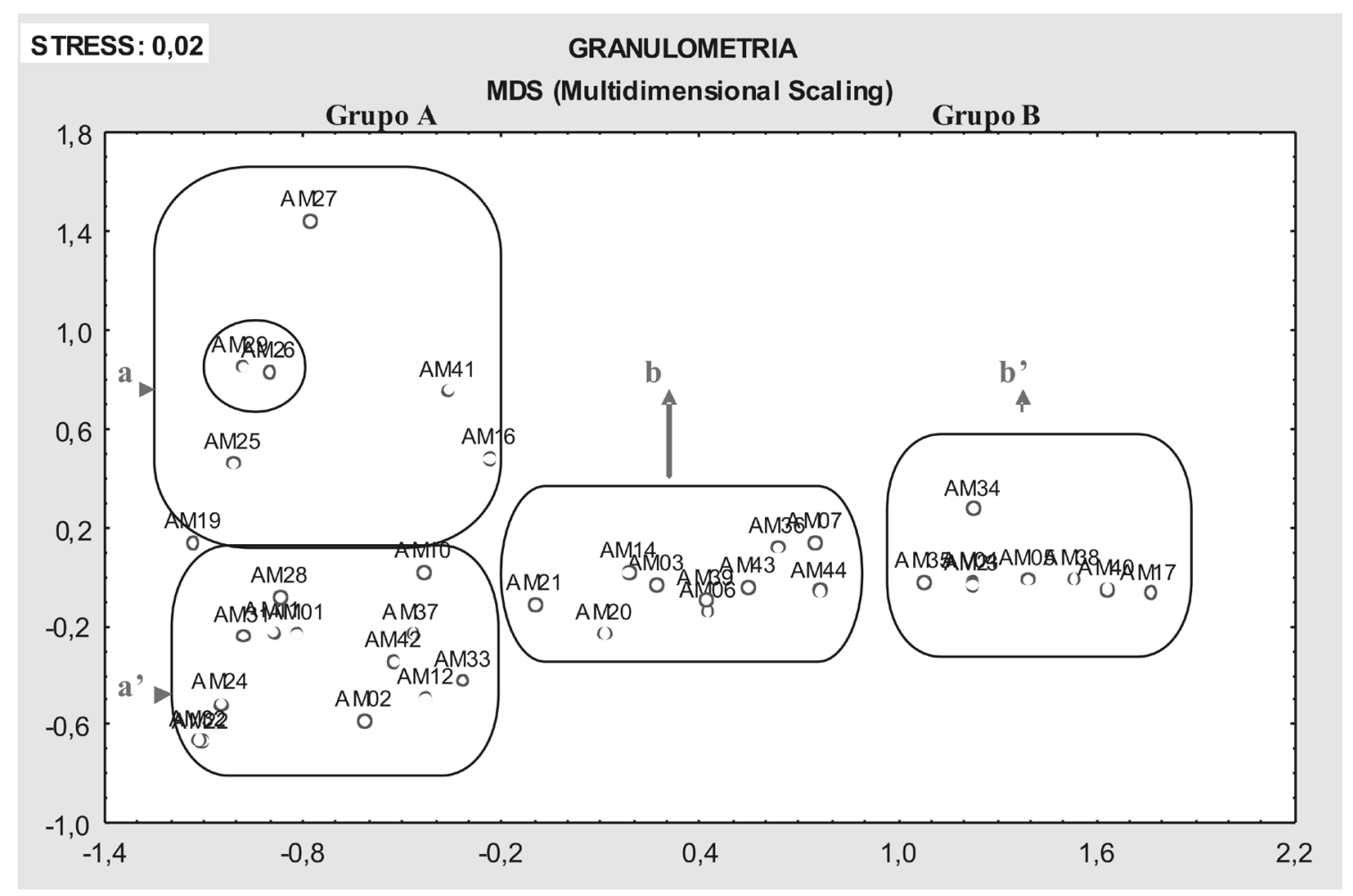

Figura 4 - Esquema representativo da ordenação apresentada pelas estações, a partir dos dados sedimentológicos (percentuais cascalho, areia e lama). Grupo $A=$ lama $<30 \%$ (Subgrupo $A=$ cascalho $>$ $20 \%$ e areia $<60 \%$; Subgrupo $A^{\prime}=$ cascalho $<20 \%$ e areia $\left.>60 \%\right)$; Grupo $B=$ lama $>30 \%$ (Subgrupo $B=$ lama $>70 \%$; Subgrupo B' = lama 30 a $70 \%$ ).

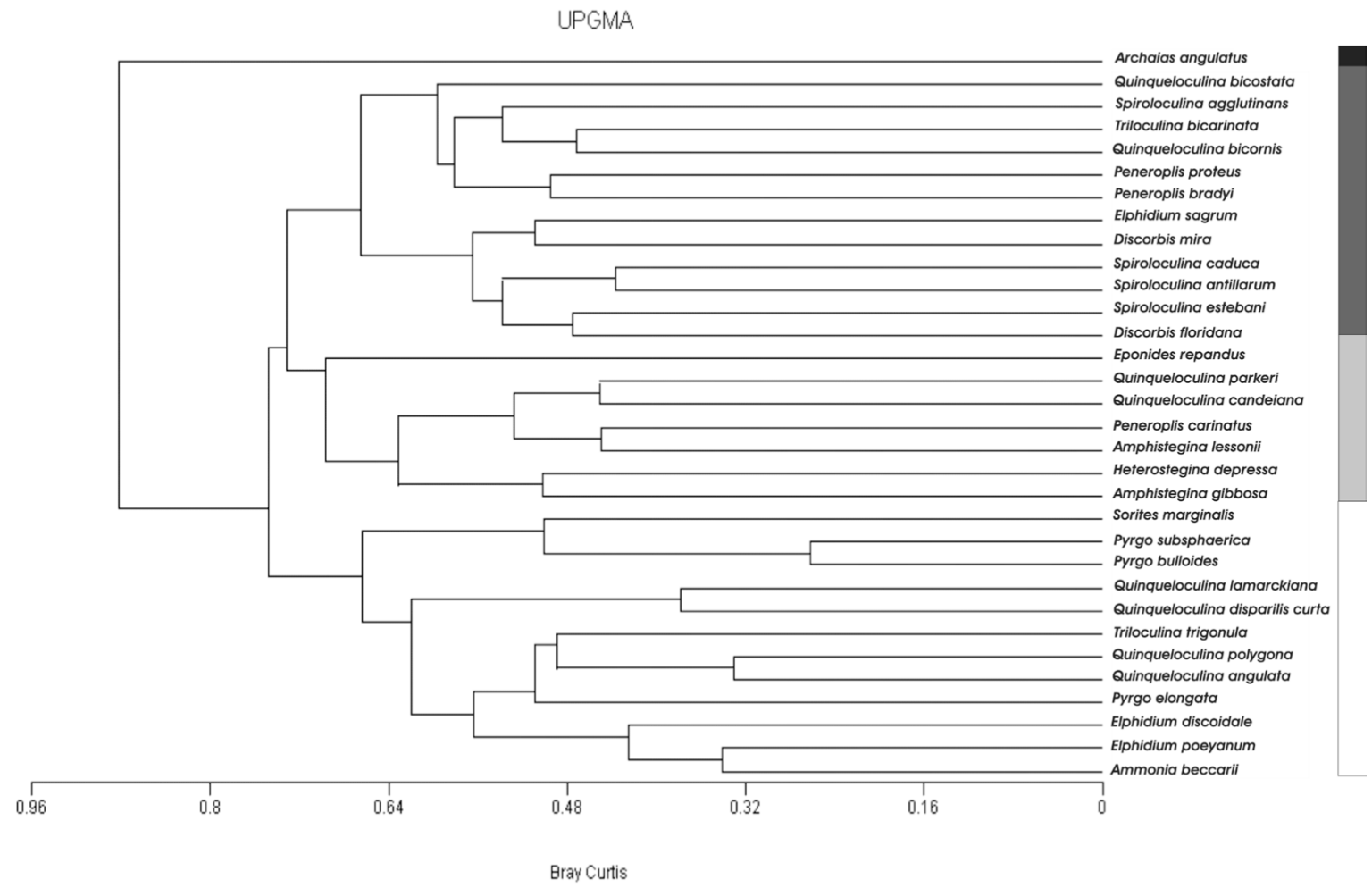
A. angulatus
Assembleia I
Assembleia II
Assembleia III

Figura 5 - Dendograma representativo do agrupamento das espécies constantes na área estudada. 
Quinqueloculina disparilis curta, Triloculina trigonula, Quinqueloculina polygona, Quinqueloculina angulata, Pyrgo elongata, Elphidium discoidale, Elphidium poeyanum e Ammonia beccarii (Fig. 5). Esta assembleia é constituída por espécies encontradas em maior abundância nas amostras correspondentes ao Grupo I da Fig. 3; portanto, amostras que, em geral, apresentam-se constituídas por sedimento com teor de lama superior a $30 \%$.

Associações amostrais O dendograma da Fig. 6 apresenta as associações obtidas entre as amostras, com base na abundância absoluta das espécies, com frequência de ocorrência superior a $50 \%$.

Podem ser observados inicialmente dois grandes grupos. O primeiro (Grupo I) é constituído por amostras cujas espécies mais representativas caracterizam a área como ambiente marinho tropical típico. As principais espécies que compõem as amostras desta associação são consideradas macroforaminíferos, estando presentes e em grande abundância em todas as amostras deste grupo, a exemplo dos táxons Amphistegina lessonii, Archaias angulatus e Peneroplis carinatus.
No segundo grupo (Grupo II) estão as amostras que apresentam baixa abundância em macroforaminíferos, constituindo-se, em geral, por espécies que apresentam carapaças menores do que os indivíduos do Grupo I e que podem ser encontradas ao longo de praticamente toda a costa brasileira, possuindo, portanto, caráter cosmopolita.

O Grupo II está dividido em dois subgrupos: A e B. As amostras que formam o subgrupo A apresentam elevada representatividade de miliolídeos e evidenciam pouquíssimos representantes da espécie Ammonia beccarii.

Por sua vez, o subgrupo B mostra-se formado por amostras que apresentam equilíbrio entre os indivíduos das subordens Miliolina e Rotaliina, estando a espécie Ammonia beccarii bem representada nas amostras deste grupo.

DISCUSSÃO Os resultados obtidos a partir da análise granulométrica do sedimento revelam que a fração cascalho é encontrada preferencialmente em áreas mais afastadas da costa, cuja composição e origem estão associadas a formações de algas calcárias, denominadas rodolitos. Bosence (1976) descreveu que

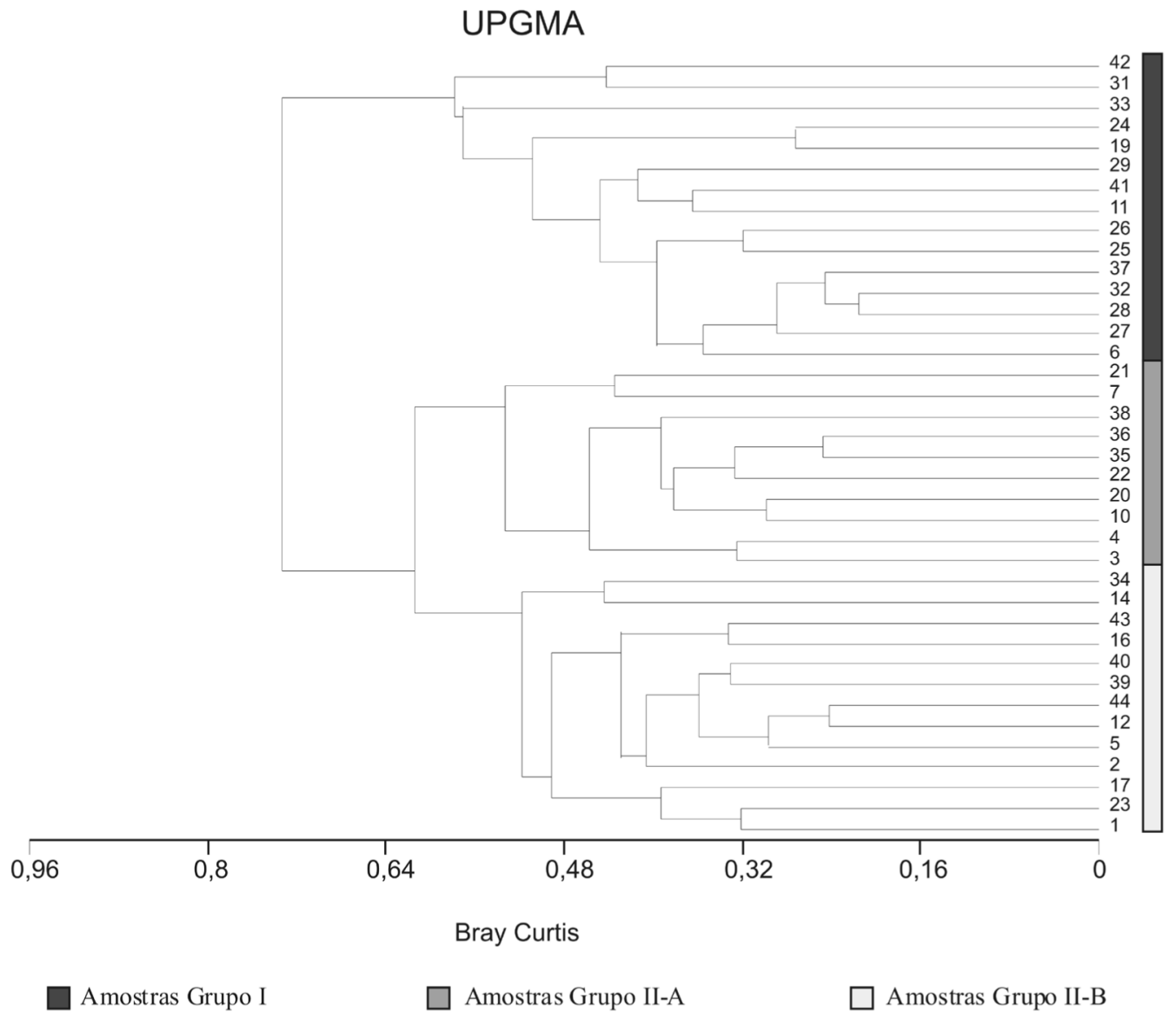

Figura 6 - Dendograma representativo do agrupamento das estações a partir da abundância absoluta das espécies constantes na área de estudo. 
estas formações calcárias na Irlanda (Mannin Bay) são restritas em função da luminosidade local, entre profundidades de 1 a 16 m. De modo geral, Dias (2001) destacou a ocorrência contínua de algas calcárias em plataforma continental brasileira a ambientes compreendidos entre a plataforma média e a externa, muitas vezes em profundidades maiores do que $50 \mathrm{~m}$.

A fração areia, predominante em toda a área de estudo, não evidencia qualquer padrão característico de distribuição. A dominância desta fração pode ser explicada pela forte atuação de processos mecânicos, uma vez que se trata de um ambiente de alta energia. Exceções são feitas às zonas recifais protegidas, nas quais é praticamente nula a ação das ondas. A fração lama, assim como a fração areia, não deixa evidente nenhum padrão de distribuição. Sua ocorrência, com teores bastante elevados em parte das amostras, e sua constituição predominantemente biogênica podem ser explicadas pelo fato de as amostragens terem sido efetuadas próximas às estruturas recifais, uma zona que apresenta inúmeros canais inter-recifais, os quais servem como armadilhas para o sedimento lamoso, impedindo que o mesmo seja transportado pelas correntes atuantes na área.

Utilizando-se uma análise comparativa entre as características sedimentológicas citadas e a microfauna de foraminíferos, pode-se notar que as relações destes organismos com o sedimento são fortes e incontestáveis, sobretudo pela associação direta entre a textura do sedimento e a distribuição da microfauna bentônica, predominante na área em estudo. Nesta perspectiva, inúmeros autores apontam que locais apresentando substrato constituído, principalmente, por granulações finas possuem maior conteúdo orgânico do que depósitos de granulação grossa (Moore 1958, Ferreira 1977 e 1978). Esse fato influencia na quantidade de vida que pode ser sustentada, ou seja, o número de espécimes em um dado volume de sedimento é mais alto nas áreas em que existe maior concentração de matéria orgânica (Phleger 1960). No entanto, analisando-se a distribuição espacial da fauna estudada, percebe-se que a heterogeneidade faunística é muito maior que as alterações encontradas nos sedimentos, as quais variaram preferencialmente entre areia e sedimentos com teores mistos de areia e lama, cuja composição apresenta-se essencialmente carbonática.

Acredita-se, portanto, que, embora as características granulométricas e composicionais do substrato exerçam influência sobre a distribuição das populações bentônicas, como já relatado por alguns autores (Boltovskoy \& Wright 1976, Kitazato 1988, Murray 1991), há outras variáveis ambientais que ajudam a justificar a distribuição observada para a fauna, por exemplo, a profundidade e a hidrodinâmica das águas. Segundo McLusky \& McIntyre (1988), na maior parte das plataformas continentais, a distribuição das espécies bentônicas está, principalmente, relacionada com as variações no substrato e com o aumento da profundidade. No Brasil, esta relação tem sido apresentada em inúmeros estudos realizados em ambientes de plataforma (Andrade 1997, Nascimento 2003, Araújo 2004, Silva 2004, Araújo \& Machado 2008a, b, c, d).

O dendograma que representa o agrupamento das espécies constantes deixa evidente que a assembleia I é formada por espécies caracterizadas na literatura pela ampla distribuição em ambientes de plataforma continental rasa, semelhantes à da área de estudo. Thomas \& Schafer (1982) associaram a ampla distribuição de espécies relacionadas neste grupo (Textularia aglutinans, Peneroplis bradyi e Peneroplis proteus), em plataformas rasas, à natureza robusta de suas testas, o que lhes confere maior resistência à hidrodinâmica do meio. Murray (1991) indicou que os gêneros Discorbis e Spiroloculina, identificados nesta assembleia, apresentam distribuição cosmopolita em ambientes de plataforma com profundidade entre 10 e $20 \mathrm{~m}$.

Aassembleia II, formada por espécies comAR elevada em amostras arenolamosas, apresenta como taxas mais abundantes, Amphistegina gibbosa, Amphistegina lessonii, Peneroplis carinatus e Heterostegina depres$s a$, espécies citadas em outros estudos pela distribuição associada a ambientes de águas rasas, quentes e de substrato arenoso e arenolamoso (Redois \& Debenay 1999, Mendes et al. 2004). A assembleia III reúne espécies de menor dimensão, mais abundantes, em amostras com teores mais elevados de lama. Estudos que avaliam a distribuição da microfauna de foraminíferos em ambientes de plataforma relatam a ampla distribuição de espécies deste grupo (Ammonia beccarii, Elphidium poeyanum, Pyrgo subsphaerica, Quinqueloculina disparilis curta e Quinqueloculina lamarckiana) a ambientes com predomínio da fração lama sobre as demais (Miao \& Thunell 1993, Nascimento 2003, Silva 2004, Araújo \& Machado 2008d, Murray 2006).

Uma análise comparativa entre o presente estudo e o trabalho realizado por Leão (1982), que analisou a distribuição dos principais grupos de foraminíferos ao longo de uma linha de amostragem 
perpendicular à costa, a partir da zona da praia até a área dos recifes externos, revelou semelhanças e diferenças na distribuição das espécies relacionadas à dos sedimentos. Nos dois trabalhos, as espécies Ammonia beccarii e Elphidium poeyanum foram encontradas preferencialmente em áreas próximas à costa, entretanto, no trabalho de Leão (1982) estas espécies foram observadas em maior abundância nas áreas ricas em sedimentos siliciclásticos, já no presente estudo, as mesmas espécies, mostram-se abundantes em áreas essencialmente carbonáticas.

Segundo Murray (1991), algumas formas calcárias, como os gêneros Ammonia, Elphidium e Nonion, são consideradas foraminíferos tolerantes a condições de redução de salinidade. Desta forma, embora não se disponha de dados de salinidade para as estações amostradas, é possível que, na área de influência dos rios Jucuruçu, Itanhaém, Caravelas e Peruípe, ocorra diminuição de salinidade, o que poderia explicar a presença da espécie Ammonia beccarii em áreas carbonáticas.

CONCLUSÕES Os resultados obtidos, por meio das análises multivariadas, revelam a relação existente entre a distribuição da microfauna de foraminíferos e a granulometria do sedimento.
$\mathrm{Na}$ área estudada existem outras variáveis ambientais, além das analisadas, que ajudam a justificar a distribuição das assembleias encontradas. Entretanto, as espécies mais abundantes de cada uma apresentam distribuição diretamente relacionada com a textura do sedimento.

Dentre as espécies mais abundantes na área, foi possível verificar que: Amphistegina lessonii e Peneroplis carinatus ocorrem preferencialmente associadas a sedimentos arenosos de composição carbonática, sendo predominantes nos recifes externos; as espécies Ammonia beccarii, Elphidium poeyanum, Pyrgo subsphaerica, Quinqueloculina disparilis curta e Q. lamarckiana apresentam-se predominantes nas amostras de sedimentos lamosos, cuja composição varia de carbonática à mista. Dentre estas espécies, Ammonia beccarii, associada a Elphidium poeyanum, predominam nas áreas recifais próximas à costa; Archaias angulatus, macroforaminífero característico de ambientes de alta energia, ocorre nas áreas inter-recifais costeiras e externas, em sedimentos mistos e carbonáti$\cos$, sem evidenciar qualquer padrão característico de distribuição em relação às características sedimentológicas da área e à profundidade em que as amostras foram coletadas.

\section{Referências}

Andrade E.J. 1997. Distribuição dos Foraminíferos Recentes na Transição Carbonatos/Siliciclástos na Região de Praia do Forte, litoral Norte do estado da Bahia. Dissertação de Mestrado, Instituto de Geociências da Universidade Federal da Bahia, Bahia, 111 p.

Araújo T.M.F. 2004. Estudo da microfauna de foraminíferos do sedimento da superfície e da subsuperfície da plataforma e do talude continentais da região norte do estado da Bahia (Salvadora Barra do Itariri). Tese de Doutorado, Instituto de Geociências da Universidade Federal da Bahia, Bahia, 525 p.

Araújo H.A.B de. 2009. Assembléias de foraminíferos indicadoras de mudanças ambientais no Complexo Recifal de Abrolhos, Bahia.Tese de Doutorado, Instituto de Geociências da Universidade Federal da Bahia, Bahia, 236 p.

Araújo T.M.F. \& Machado A.J.M. 2008a. Eventos bioestratigráficos, paleoclimáticos e paleobatimétricos do talude continental superior da Bahia, evidenciados através do estudo da fauna dos Foraminíferos. Gravel, 6(1):27-45.

Araújo T.M.F. \& Machado A.J.M. 2008b. Foraminíferos da subsuperfície do talude continental superior do norte da Bahia, Brasil. Revista de Geologia, 21(1):49-77.
Araújo T.M.F. \& Machado A.J.M. 2008c. Analise sedimentar e micropaleotológica (Foraminíferos) de Seções Quaternárias do Talude Continental Superior do Norte da Bahia, Brasil. Revista Pesquisas em Geociências, 35(2):97-113.

Araújo T.M.F. \& Machado A.J.M. 2008d. Benthic foraminifera associated with the South Bahia Coral Reefs, Brazil. Journal of Foraminiferal Research, 28(1):23-38.

Barker R.W. 1960. Taxonomic notes. Oklahoma, Society of Economic Paleontologists and Mineralogists, $238 \mathrm{p}$.

Bergue C.T. \& Coimbra J.C. 2008. Late Pleistocene and Holocene bathyal ostracodes from the Santos Basin, southeastern Brazil. Palaeontographica, 285:101-144.

Bock W.D. 1971. The use of Foraminifera as indicators of subsidence in the Caribbean. (Abs) In: Sixth Carib. Geol. Conf., 28 p.

Boltovskoy E. \& Wright R. 1976. Recent Foraminifera. Dr. W. Junk, The Hague, Buenos Aires, 515 p.

Boltovskoy E., Giussani G., Watanabe S., Wright R. 1980. Atlas of benthic shelf foraminifera of the southwest Atlantic. Boston, Dr. W. Junk by Publishers, $147 \mathrm{p}$. 
Boltovskoy E., Scott D.B., Medioli F.S. 1991. Morphological variations of benthic foraminiferal test in response to changes in ecological parameters: a review. Journal of Paleontology, 65(2):175-185.

Bosence D. 1976. Ecological studies on two unattached coralline algae from western Ireland. Paleontology, 19(2):365-395.

Bruno R.L.M. 2008. Avaliação das assembléias de foraminíferos na plataforma de transição carbonatosiliciclasto, região de Ilhéus, Bahia. Dissertação de Mestrado, Instituto de Geociências da Universidade Federal da Bahia, Bahia, 121 p.

Clarke K.R. \& Warwick R.M. 1994. Change in Marine Communities: An Approach to Statistical Analysis and Interpretation. Plymouth, Plymouth marine Laboratory, $144 \mathrm{p}$.

Closs D. \& Barberena M.C. 1960. Foraminíferos recentes da Praia da Barra (Salvador, Bahia). Escola Geológica de Porto Alegre, 6:1-50.

Dajoz R. 1983. Ecologia geral. 4. ed., Petrópolis, Vozes, $475 \mathrm{p}$.

Debenay J.P., Pawlowski J., Decrouez D. 1996. Lês foraminifères actuels. Masson, Paris, $329 \mathrm{p}$.

Debenay J.P. 1988. Foraminifera large than $0,5 \mathrm{~mm}$ in the southwestern Lagoon of New Caledonia: Distribution related to abiotic properties. Journal of Foraminiferal Research, 8(2):158-175.

Dias G.T.M. 2001. Granulados bioclásticos - algas calcárias. Brazilian Journal of Geophysics, 18(3):307-318.

Ferreira M.T.G.M. 1977. Foraminíferos da zona de intermarés de Itapuã - Salvador, Bahia. Dissertação de Mestrado, Instituto de Geociências da Universidade Federal da Bahia, Bahia, 186 p.

Ferreira A.B. 1978. Planaltos e montanhas do Norte da Beira. Estudo de geomorfologia. Memórias do Centro de Estudos Geográficos, Lisboa, 374 p.

Kitazato H. 1988. Ecology of Benthic Foraminífera in the Tidal Zone of a Rocky Shore. Revue de Paléobiologie, (2):815-825.

Leão Z.M.A.N. 1982. Morphology, geology and developmental history of the southermost coral reefs of Western Atlantic, Abrolhos Bank, Brazil. Ph.D. Dissertation, Rosenstiel School of Marine and Atmospheric Science, University of Miami, Florida, USA, $218 \mathrm{p}$.

Leão Z.M.A.N. 1983. Abrolhos, o refúgio pleistocênico de uma fauna terciária de corais. Revista de Biologia e Ciências da Terra, 8:22-24.

Leão Z.M.A.N. 1996. The coral reefs of Bahia: morphology, distribution and the major environmental impacts. Anais da Academia Brasileira de Ciências, 68(3):439-452.

Leão Z.M.A.N., Dutra L.X.C., Spanó S. 2001. The Characteristic of Bottom Sediments in Marine Assessment Program Survey of the Abrolhos Bank, Brazil. Conservation International of Brazil. Unpublished Technical report, $66 \mathrm{p}$.
Leão Z.M.A.N., Kikuchi, R.K.P, Oliveira, D.M.O. 2008. Branqueamento de corais nos recifes da Bahia e sua relação com eventos de anomalias térmicas nas águas superficiais do oceano. Biota Neotropica, 8(3):69-82.

Leipnitz I.I. 1991. Ocorrência das Ammodiscaceas e Lituolaceas em sedimentos recentes da plataforma continental e do talude norte do Brasil. (desembocadura do Rio amazonas ao Cabo Orange). Acta Geológica Leopoldensia, 33(14):129-152.

Leipnitz I.I., Leipnitz B., Beckel J. 1992. Ocorrência de miliolaceas am sedimentos recentes da plataforma continental e do talude norte do Brasil. (desembocadura do Rio amazonas ao Cabo Orange). Acta Geológica Leopoldensia, 33(15):5-30.

Levy A., Mathieu R., Poignant A., Rosset-Moulinier M., Ubaldo M.L., Lebreiro S. 1995. Foraminifers actuals de la marge cantinentale portugaise - inventaire et distribution. Memórias do Instituto Geológico e Mineiro, 32, Lisboa, 97 p.

Loeblich A. R. Jr. \& Tappan H. 1988. Foraminiferal genera and their classification. Van Nostrand Reinhold Ed. New York, 970 p.

Machado A.J. 1981. Coloração dos Foraminíferos Bentônicos e Planctônicos dos Sedimentos da Margem Continental Norte Brasileira. In: XXXIII Reunião Anual Da Sociedade Brasileira Para O Progresso Da Ciência - SBPC, SalvadorBA. Suplemento de ciências e cultura, 33, 547 p.

McLusky D.S. \& McIntyre A.D. 1988. Characteristics of the Benthic fauna. In: Postma H., Zijlstra J. J. (eds) Continental shelves of the world, 27, Elsevier, Amsterdam, p. 131-154.

Mendes I., Gonzales R., Dias J.M.A., Lobo F., Martins V. 2004. Factors influencing recent benthic foraminifera distribution on the Guadiana shelf (Southwestern Iberia). Marine Micropaleontology, 51:171-192.

Miao Q. \& Thunell R.C. 1993. Recent deep-sea benthic foraminiferal distributions in the south China and Sulu Seas. Marine Micropaleontology, 22:1-32.

Moore H.B. 1958. Marine Ecology. New York, Wiley \& Sons. 493 p.

Moraes S.S. 2001. Interpretações da hidrodinâmica e dos tipos de transporte a partir de análises sedimentológicas e do estudo dos foraminíferos recentes dos recifes costeiros da Praia do Forte e de Itacimirim, litoral norte do Estado da Bahia. Salvador. Dissertação de Mestrado, Instituto de Geociências da Universidade Federal da Bahia, Bahia, 64 p.

Moraes S.S. 2006. Distribuição espacial e tafonomia de foraminíferos na plataforma continental da região norte da costa do dendê (foz do Rio Jequiriçá à Ponta dos Castelhanos), Bahia. Tese de Doutorado, Instituto de Geociências, Universidade Federal da Bahia, Bahia, 102 p.

Murray J.W. 1991. Ecology and Palaeoecology of Benthic Foraminifera. New York, Longman Scientific \& Technical, 397 p.

Murray J. W. 2006. Ecology and Applications of Benthic Foraminifera. New York, Melbourne: Cambridge University Press, 426 p. 
Nascimento H.A. 2003. Análise da Fauna de Foraminíferos Associada aos Recifes do Extremo Sul do Estado da Bahia (Corumbau a Nova Viçosa). Dissertação de Mestrado, Instituto de Geociências, Universidade Federal da Bahia, Bahia, 145 p.

Ocean Wheater. 2008. Brazil Offshore Meteorological and Oceanography Study. NOAA - Sattelite and Information Service.

Olavo G., Costa P.A.S., Martins A.G. 2007. Estrutura de comunidades de peixes recifais na plataforma externa e talude superior da costa central brasileira: diversidade e distribuição batimétrica. In: Costa P.A.S., Olavo G., Martins A.S. (eds.) Biodiversidade da fauna marinha profunda na costa central brasileira. Rio de Janeiro, Museu Nacional. p. 15-43 (Série Livros n. 24).

Phleger F.B. 1960. Ecology and distribution of Recent foraminifera. Johns Hopkins, Baltimore, Md. 287 p.

Redois F. \& Debenay J.P. 1999. Re'partition des foraminifers benthiques actuels sur le plateau continental sénégalais ai sud de Dakar. Oceanologica Acta, 22(2):215-232.

Rossi A.R. 1999. Foraminíferos quaternários do Arquipélago de Fernando de Noronha: taxonomia, ecologia, distribuição batimétrica e faciológica. Dissertação de Mestrado, Universidade Vale do Rio dos Sinos, Porto Alegre, 141 p.

SEI 1998. Análise dos atributos climáticos do Estado da Bahia. Salvador, Série Estudos e Pesquisas, 38, 85 p.
Sen Gupta B.K. 1999. Systematics of Modern Foraminífera. In: Sen Gupta B.K (ed.) Modern Foraminifera. Kluwer Academic Publishers, London, p. 3-36.

Silva S.S.F. 2004. Caracterização da fauna de foraminíferos presentes no sedimento superficial do fundo da plataforma continental da costa atlântica de Salvador. Dissertação de Mestrado, Instituto de Geociências da Universidade Federal da Bahia, Bahia, 82p.

Thomas F.C. \& Schafer C.T. 1982. Distribution and transport of some common foraminiferal species in the Minas Basin, Eastern Canada. Journal of Foraminiferal Research, 12(1):71-90.

Tinoco I.M. 1955. Foraminíferos recentes de cabo Frio, Estado do Rio de Janeiro. Rio de Janeiro, Instituto Brasileiro de Geografia e Estatística, 43 p., Boletim 159.

Tinoco I.M. 1958. Foraminíferos quaternários de Olinda, Estado de Pernambuco. Rio de janeiro, Departamento Nacional de Produção Mineral do Ministério da Agricultura, 61 p., Monografia 14.

Tinoco I.M. 1989. Introdução ao estudo dos componentes bióticos dos sedimentos marinhos recentes. Recife, Editora Universitária da UFPE, 219 p.

Valentin J.L. 2000. Ecologia numérica: uma introdução à análise multivariada de dados ecológicos. Jean Louis Valentin - Rio de Janeiro, Interciência, 117 p.

Manuscrito ID 18707

Recebido em: 23/09/2010

Aprovado em: 23/04/2012 